\title{
Improving Data Confidentiality and Integrity for Data Aggregation in Wireless Sensor Networks
}

\author{
Rabindra BISTA $^{\dagger}$, Yong-Ki KIM ${ }^{\dagger}$, Myoung-Seon SONG ${ }^{\dagger}$, Nonmembers, and Jae-Woo CHANG ${ }^{\dagger a)}$, Member
}

\begin{abstract}
SUMMARY Since wireless sensor networks (WSNs) are resourcesconstrained, it is very essential to gather data efficiently from the WSNs so that their life can be prolonged. Data aggregation can conserve a significant amount of energy by minimizing transmission cost in terms of the number of data packets. Many applications require privacy and integrity protection of the sampled data while they travel from the source sensor nodes to a data collecting device, say a query server. However, the existing schemes suffer from high communication cost, high computation cost and data propagation delay. To resolve the problems, in this paper, we propose a new and efficient integrity protecting sensitive data aggregation scheme for WSNs. Our scheme makes use of the additive property of complex numbers to achieve sensitive data aggregation with protecting data integrity. With simulation results, we show that our scheme is much more efficient in terms of both communication and computation overheads, integrity checking and data propagation delay than the existing schemes for protecting integrity and privacy preserving data aggregation in WSNs.

key words: wireless sensor network, data aggregation, data integrity, data privacy, signature
\end{abstract}

\section{Introduction}

In WSNs [1], data from sensor nodes are correlated in terms of time and space. Therefore, transmitting only the required and partially processed data is more meaningful than sending a large amount of raw data. In general, sending raw data causes energy blindness because duplicated message are sent to the same node, called implosion, as well as neighboring nodes receive duplicate messages if two nodes share the same observing region, called overlapping. So, data aggregation which combines data coming from many sensor nodes has been actively researched in recent years. An extension of this approach is in-network aggregation which aggregates data progressively as data are passed through the network [2]-[4], [9], [14]. In-network data aggregation can reduce the number of data transmissions and the number of nodes involved in gathering data from a WSN.

Another issue of WSNs is how to preserve sensitive measurements of everyday life where data privacy becomes an important aspect. In many scenarios, confidentiality of transported data can be considered critical, for instance, data from sensors might measure patients' health information such as heartbeat and blood pressure details. Also, a future application might measure household details such as power and water usage, computing average trends and making lo-

Manuscript received March 15, 2011.

Manuscript revised June 29, 2011.

†The authors are with the Dept. of Computer Eng., Chonbuk National Univ., Chonju, Chonbuk, 561-756, Korea.

a) E-mail: jwchang@chonbuk.ac.kr

DOI: 10.1587/transinf.E95.D.67 cal recommendations. Since sensitive data are transported wirelessly among sensor nodes, they are typically prone to interception and eavesdropping. So, maintaining data privacy of a sensor node even from other trusted participating sensor nodes of the WSN is mandatory. As a result, even though private data are overheard and decrypted by adversaries or other trusted participating sensor nodes, it can prevent to recover sensitive information of a sensor node [5], [15], [16].

The other issue is data integrity [6]-[8], [17]. In communication, data integrity is simply defined as maintaining consistency and correctness of message (message without modification by adversaries). In other words, this is about how to ensure, by the sink node, the received data is not altered in transit either by an adversary or by noise. Data pollution due to the noise is an unintentional process and it can be handled by using some existing mechanisms like Cyclic Redundancy Checking (CRC). Hence, the integrity checking due to the unintentional data pollution is out of the scope of this research. But, the mechanisms like CRC are unable to cope with the intentional data pollution by an adversary (compromised node) because the adversary can generate the same CRC of the source node after modifying the data. As data aggregation result is used for making critical decisions, the aggregation result must be verified before accepting it. For this reason, it is required to design a scheme for WSNs which can ensure the aggregated result has not been polluted (manipulation of data by an adversary) on the way to the query server and this issue is one of the main focuses of our research.

Since data privacy and integrity protection processes consume a significant amount of precious resource (i.e., limited power) of sensor nodes they shorten the lifetime of the WSN. Therefore, it is necessary to devise a light-weight scheme which can achieve data privacy and integrity protection efficiently. But, the existing work needs much resource of sensor nodes due to generation of unnecessary messages in the network. For this reason, in this paper, we propose a new and resource efficient scheme that can aggregate sensitive data protecting data integrity in WSNs. Our scheme utilizes complex numbers, which is an algebraic expression and can use arithmetic operations, such as addition (+), to aggregate and hide data (for data privacy) from other sensor nodes and adversaries during transmissions to the data sink. In our scheme, the real unit of a complex number is used for concealing sampled data whereas the imaginary unit is used for providing data integrity checking. Thus, our scheme not 
only prevents recovering sensitive information even though private data are overheard and decrypted by adversaries or other trusted participating sensor nodes but also provides data integrity checking. For data security, our scheme can be built on the top of the existing secure communication protocols like [12]. Moreover, our propose scheme is a general approach so that it can be applied to any type of WSN in terms of network topology.

The rest of the paper is organized as follows. In Sect. 2, we present some related work. Section 3 describes our integrity protecting sensitive (private) data aggregation scheme in detail. Simulation results are shown in Sect. 4. Along with some future research directions, we finally conclude our work in Sect. 5.

\section{Related Work}

The authors of Privacy-preserving Data Aggregation (PDA) protocol [5] proposed two schemes to handle data aggregation by protecting data privacy for WSNs. They are the Cluster-based Private Data Aggregation (CPDA) and SliceMix-AggRegaTe (SMART) schemes. Furthermore, there are other work by Conti et al. [16] and Feng et al. [15] to address privacy-preserving data aggregation for WSNs. Although the work [5], [15], [16] can prevent to recover sensitive information when the neighboring nodes overhear and decrypt the private data, these protocols are lack of supporting data integrity feature.

Recently, He et al. proposed iPDA [7] and iCPDA [17] schemes for WSNs to support integrity checking by extending their previous protocols SMART and CPDA respectively. In the iPDA scheme, they protect data integrity by designing node disjoint two aggregation trees rooted at the query server where each node belongs to a single aggregation tree. For data privacy preservation, it utilizes data slicing and assembling technique of the SMART. In this technique, first, each sensor node randomly selects a set of $L$ number of sensor nodes, within hop $h$, from each of the aggregation tree. Every sensor node slices its private data randomly into $L$ pieces and $L-1$ pieces are encrypted and sent to the randomly selected sensor nodes of the aggregation tree keeping one piece at the same sensor node. In this process, a single sensor node receives multiple slices from multiple sensor nodes. The same process is independently done for each sensor node using another aggregation tree. Then, all the sensor nodes which received data slices from multiple sensor nodes decrypt the slices using their shared keys and sum the received data slices including its own. After that, each sensor node sends the sum value to its parent from the respective aggregation tree. In the same way, the sum data from another set of sensor nodes are transmitted to the query server through another aggregation tree. In the end, the aggregated data from two node-disjoint aggregation trees reach to the base station where the aggregated data from both aggregation trees are compared. If the difference of the aggregated data from the two aggregation trees doesn't deviate from the predefined threshold value the query server accepts the aggregation result, otherwise, it rejects the aggregated result by considering them as polluted data.

However, there are some shortcomings in the iPDA. First of all, it is impractical to compare aggregated values of two node-disjoint aggregation trees to check data integrity because a WSN are not always reliable, it can not be expected that all nodes reply to all requests. Secondly, during protecting data privacy it generates high traffics in the WSN. As a result, communication cost is significantly increased in the iPDA. Thirdly, to secure communication channel from adversaries, all sensor nodes use secret keys to encrypt their all data slices before sending to their respective $2(L-1)$ number of sensor nodes. So, every sensor node has computation overhead of decrypting all the slices they received before aggregating them. Encryption-decryption is expensive operation for resources-constrained sensor nodes. Therefore, computation cost is also high in the iPDA. Fourthly, slicing and assembling technique can only tolerate the collusion of up to a certain threshold number of sensor nodes, i.e., the sum of out-degree and in-degree minus one. If the number of colluding sensor nodes exceeds the threshold, the sensor nodes may collaboratively reveal the private information of some of the others. Although the threshold can be raised by increasing the number of slices, it will further increase communication overhead. Fifthly, since each sensor node on average has to transmit and receive five to six messages the data propagation delay is very high in iPDA scheme. Finally, the iPDA, which use slicing and assembling technique, has limited scope in terms of supporting network topology. The reason is that the iPDA is not suitable to a scenario that uses clustered network topology where only the cluster leader is considered to process data.

In the iCPDA, three rounds of interactions are required: Firstly, each node sends a seed to other cluster members. Next, each node hides its sensory data via the received seeds and sends the hidden sensory data to each cluster member. Then, each node adds its own hidden data to the received hidden data, and sends the calculated results to its cluster head which calculates the aggregation results via inverse and multiplication of matrix. To enforce data integrity, cluster members check the transmitted aggregated data of the cluster head. There are some disadvantages of iCPDA. Firstly, the communication overhead of iCPDA increases quadratically with the cluster size. Secondly, the computational overhead of CPDA increases quickly with the increase of the cluster size which introduces large matrix, whereas lower cluster size introduces lower privacy-preserving efficacy. Thirdly, the three rounds of interactions introduce data propagation delay. Finally, iCPDA is suitable only for cluster topology.

Both iPDA and ICPDA support very weak data integrity checking because if any node modifies its sampled value 30 to 300 and uses the value 300 for aggregation process none of both methods can detects such misbehavior in the network. Hence, in this paper, we propose a new, efficient (in terms of communication overhead and data 
propagation delay) and general (in terms of supporting network topology) scheme in order to support data privacy and achieve integrity assurance in data aggregation for WSNs. Our scheme is based on the algebraic properties of the complex numbers and it not only ensures that no trend about sensitive data of a sensor node is released to any other nodes and adversaries but also provides data integrity checking of the aggregated value of sensor data.

\section{Integrity-Protecting Sensitive Data Aggregation Protocol}

To overcome previously mentioned shortcomings of the iPDA, in this section, we propose a new and efficient mechanism for preserving data privacy during their aggregation in WSNs. At the same time, it can check data integrity of the aggregated data at both data aggregator and the sink node/query server. Our scheme is a general approach because it can be applied to any network topology. Our scheme exploits complex numbers by using their additive property to aggregate sensor data in WSNs. Out of two parts of a complex number, the real part is used to hide the sampled data of a sensor node from its neighboring nodes and adversaries whereas the imaginary part is used for data integrity checking at both data aggregator and the sink node. Before transmitting data to a parent node, every sensor node transforms its sampled data into a complex number form by combining the sampled data with a unique private seed and appending an imaginary unit (a real number adjoined with $i$ ) with the modified sampled data. For this, first, the sampled value is mingle with a real number (private seed) and then the result appends another real number with $i$ to give the value a complex number form $(C=a+b i)$. The real number with $i$ is the absolute difference value of the previous sample data and the current sample data of a node. (Note: during network deployment, a Master Device (MD) [12] securely provides a unique real number as a seed to every sensor node of the WSN after establishing a pairwise secret key with them. Since the MD is offline and not an online server, it shares this information only with the query server for future reference. Thus, the seed of each sensor node is private in the network). Data can be aggregated in upper hierarchy levels during their transmissions to the query server by using algebraic properties of complex numbers. In particular, we apply the additive property of complex number for data aggregation. This is because, like in [5], we also focus on additive aggregation function (Sum). We know that other aggregation functions, such as Average, Count, Variance, Standard Deviation and any other Moment of the measured data, can be reduced to the additive aggregation function Sum [10].

\subsection{Network Model and Background}

We assume a general aggregated multi-hop WSN model as shown in Fig. 1. The network model has the following properties. First, either a resource-constrained query server,

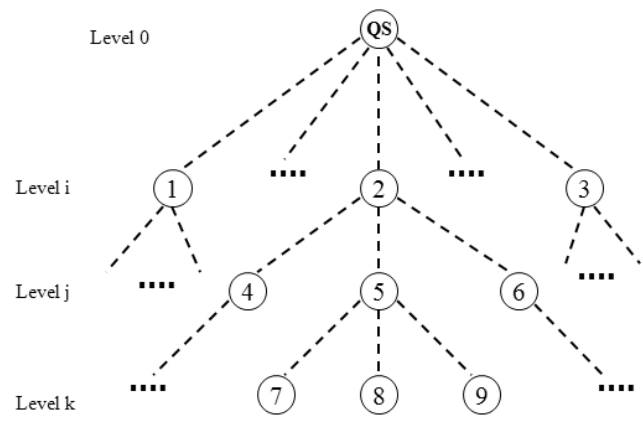

Fig. 1 Multi-hop aggregation WSN with a query server (QS) at the top.

for example a MICA mote [13], or a powerful query server (QS), for example a laptop, can be the root of the network topology and it may be located anywhere in the network. Secondly, a large number of resource-constrained sensor nodes (MICA motes) are deployed uniformly in the network area and they are arranged in different levels based on their hop counts from the QS. Thirdly, each sensor node has the capabilities of sensing, aggregating and forwarding sampled data and it can send fixed-length data packet to the QS periodically. Finally, the sensor nodes can switch into a sleep mode or a low power mode in order to preserve their energy when they do not need to receive or send data as shown in [4] (Madden et al., 2005). The query server can use any efficient tree construction algorithm such as the TAG [3] and Semantic Routing Tree [4] for disseminating an aggregation query to get aggregated value of all sensor readings. We also assume that all sensor nodes share two types of key [14]. The first type is a pairwise secret key with the MD to be a trusted member of a WSN. The second type is symmetric pairwise keys with those sensor nodes lying on their aggregation tree for secure transmission channel. It has been shown that the work presented in [14] has an efficient: scaling with $O(\log N)$, where $N$ is the number of sensor nodes, behavior in terms of memory consumption and radio transmissions by guaranteeing a secure key establishment not only with a probability $p<1$. Some definitions related to our scheme are given below.

Definition 1. A complex number $C$ is an extension of the real numbers obtained by adjoining an imaginary unit, denoted by $i$, which satisfies: $i^{2}=-1$ i.e., $i=\sqrt{-1}$.

For example, if we square $20 i$ then the result is: $(20 i)^{2}=$ $(20)^{2} * i^{2}=400 *(-1)=-400$.

Definition 2. Every complex number $C$ can be written in the form $C=a+b i$, where $a$ and $b$ are real numbers called the real part and the imaginary part of the complex number respectively.

For example, in $C=35+60 i, 30$ and $60 i$ are real and imaginary units respectively.

Definition 3. Complex numbers can be added, subtracted, multiplied, and divided by formally applying associative, commutative and distributed laws of algebra. 
For example, if we apply arithmetic operator addition $(+)$ to two complex numbers $C_{1}=20+50 i$ and $C_{2}=40+35 i$ then the result is $C=60+85 i$.

Definition 4. The sampled data by a sensor node is said to be masked value when it is combined with a private real number.

For example, if temperature reading by a sensor node is 25 and 111 is a real number then 136 is the masked value.

Definition 5. A masked value is said to be customized data when an imaginary unit is adjoined with it and the customized data has a complex number form.

For example, if 136 is a masked value and $2 i$ is an imaginary unit then $136+2 i$ is a customized data which is a complex number.

\subsection{Prerequisite}

In this section, we briefly review an efficient key establishment scheme proposed in [12], which we adopt to our method for achieving security. There are two main steps involved in [12] to establish keys. The first step is to generate pairwise keys between a special device, called Master Device (MD), and all sensor nodes of a WSN. By using the $\mathrm{MD}$, a pairwise key is distributed to every sensor node by one-to-one communication between the MD and a sensor node. As a result, every node that has ever joined the network successfully possesses a pairwise secret key with MD and recognizes other sensor nodes. Moreover, a new node to join the WSN has to be paired with MD to properly enter the network.

The second step is to generate a symmetric key that is shared by other sensor nodes on its aggregation tree. By using the information of pairwise key and node IDs, every sensor node can generate a symmetric key $\mathrm{K}$. The symmetric key shared between node ' $\mathrm{x}$ ' and node ' $\mathrm{y}$ ' is called $\mathrm{K}_{\mathrm{x}, \mathrm{y}}$ (symmetric means $K_{x, y}=K_{y, x}$ ). The main idea is to split a key $\mathrm{K}$ perfectly into two key shares $\mathrm{K}_{1}$ and $\mathrm{K}_{2}$ by choosing a random number $r$ of the same size as $\mathrm{K}$ and computing

$$
\begin{aligned}
& \mathrm{K}_{1}=\mathrm{r} \\
& \mathrm{K}_{2}=\mathrm{K} \oplus \mathrm{r} \text {, where } \oplus \text { means XOR. }
\end{aligned}
$$

Moreover, in the same manner, both $\mathrm{K}_{1}$ and $\mathrm{K}_{2}$ can be further split at the key receiving nodes and distributed to other sensor nodes to deal with multiple number of compromised sensor nodes. The key splitting process continues if it is required. For instance, to deal with $\mathrm{k}$ number of compromised sensor nodes in a WSN, the symmetric key is split into k+1 shares (entries) and distributed to different sensor nodes so that at least one non-compromised sensor node can ensure security for the whole key. As shown in [24], the key establishment can be achieved in $\mathrm{O}\left(\mathrm{n}^{2}\right)$ time complexity, where $\mathrm{n}$ is the number of key shares.

These $K_{1}$ and $K_{2}$ are distributed to the initial random nodes. The restoration of $\mathrm{K}=\mathrm{K}_{1} \oplus \mathrm{K}_{2}$ is possible only when both $K_{1}$ and $K_{2}$ are known to the same node. Knowledge of only $\mathrm{K}_{1}$ or $\mathrm{K}_{2}$ will not reveal anything about K [21], [24]. For example, the work [24] assumes that $P=\left\{p_{1}, p_{2}, \ldots, p_{n}\right\}$ is the set of participants corresponding to the vertices of graph $\mathrm{G}_{\mathrm{F}}$ on finite field $\mathrm{F}$ and that the secret key $\mathrm{K}=\left(\mathrm{K}_{1}, \mathrm{~K}_{2}\right)$ is taken randomly from $\mathrm{G}_{\mathrm{F}}\left(q^{2}\right)$, where $\mathrm{q}$ is a prime and $\mathrm{q} \geq \mathrm{n}$. Let $\mathrm{f}(\mathrm{x})=\left(\mathrm{K}_{2} \mathrm{x}+\mathrm{K}_{1}\right) \bmod q \cdot y_{i}$ is computed from $f(x)$ as follows:

$$
y_{i}=f(i-1) \bmod q, \text { for } i=1, \ldots, n .
$$

It is clear that $\mathrm{f}(\mathrm{x})$ can be determined uniquely when $\mathrm{y}_{\mathrm{i}}$ and $\mathrm{y}_{\mathrm{j}}$, for $\mathrm{i} \neq \mathrm{j}$, are given. Therefore, he/she can reveal K only if he/she can get two or more shares of K. In this way, the safety of the encryption level can be proved.

It has been shown that the work presented in [12] outperforms the work presented in [22]. This is because [12] has an efficient scaling with $\mathrm{O}(\log \mathrm{n})$, where $\mathrm{n}$ the number of node, in terms of memory consumption and radio transmissions for guaranteeing secure key establishment. On the other hand, [22] consumes memory and radio energy with $\mathrm{O}(\mathrm{n})$ for providing security with a probability $\mathrm{p}<1$.

For encryption and decryption methods, we use the RC5 encryption algorithm[23] which is supported by TinyOS [20]. RC5 is a block-cipher with both a two-word input (plaintext) block and a two-word (ciphertext) output block, which are denoted by A and B, respectively. The encryption algorithm in pseudo-code is as follow:

$$
\begin{aligned}
& A=A+S[0] ; B=B+S[1] ; \\
& \text { for } i=1 \text { to } r \text { do } \\
& \qquad \begin{array}{l}
A=((A \oplus B)<<<B)+S[2 * i] ; \\
B=((B \oplus A)<<<A)+S[2 * i+1] ;
\end{array}
\end{aligned}
$$

The decryption algorithm in pseudo-code is as follow:

$$
\begin{aligned}
& \text { for } i=r \text { downto } 1 \text { do } \\
& \qquad \begin{array}{l}
B=((B-S[2 * i+1])>>>A) \oplus A ; \\
A=((A-S[2 * i])>>>B) \oplus B ; \\
B=B-S[1] ; A=A-S[0] ;
\end{array}
\end{aligned}
$$

Here, $\mathrm{S}$ is an expanded key table, $\mathrm{S}[0 \ldots 2 \mathrm{r}+1]$, consisting of $2(\mathrm{r}+1) w$-bit words, where $r$ is the number of rounds, and $\mathrm{S}$ is derived from the user's supplied secret key. In the encryption algorithm, "+" means a modulo- $2^{\mathrm{w}}$ addition operator while in the decryption algorithm, "-" means the inverse operator of "+". $\oplus$ denotes a bit-wise exclusive OR operator. A left-rotation of words and a right-rotation of words are denoted by $<<<$ and $>>>$, respectively.

It is shown from our experiment that the time overhead of both encryption and decryption processes can be negligible. This is because the time overhead of both encryption and decryption requires 2.0 microseconds, which is only about $5 \%$ of the whole query processing overhead.

3.3 Algorithm for SUM Aggregation Function with Privacy-Preservation and Checking Data Integrity

A WSN is resources-constrained in terms of power supply 


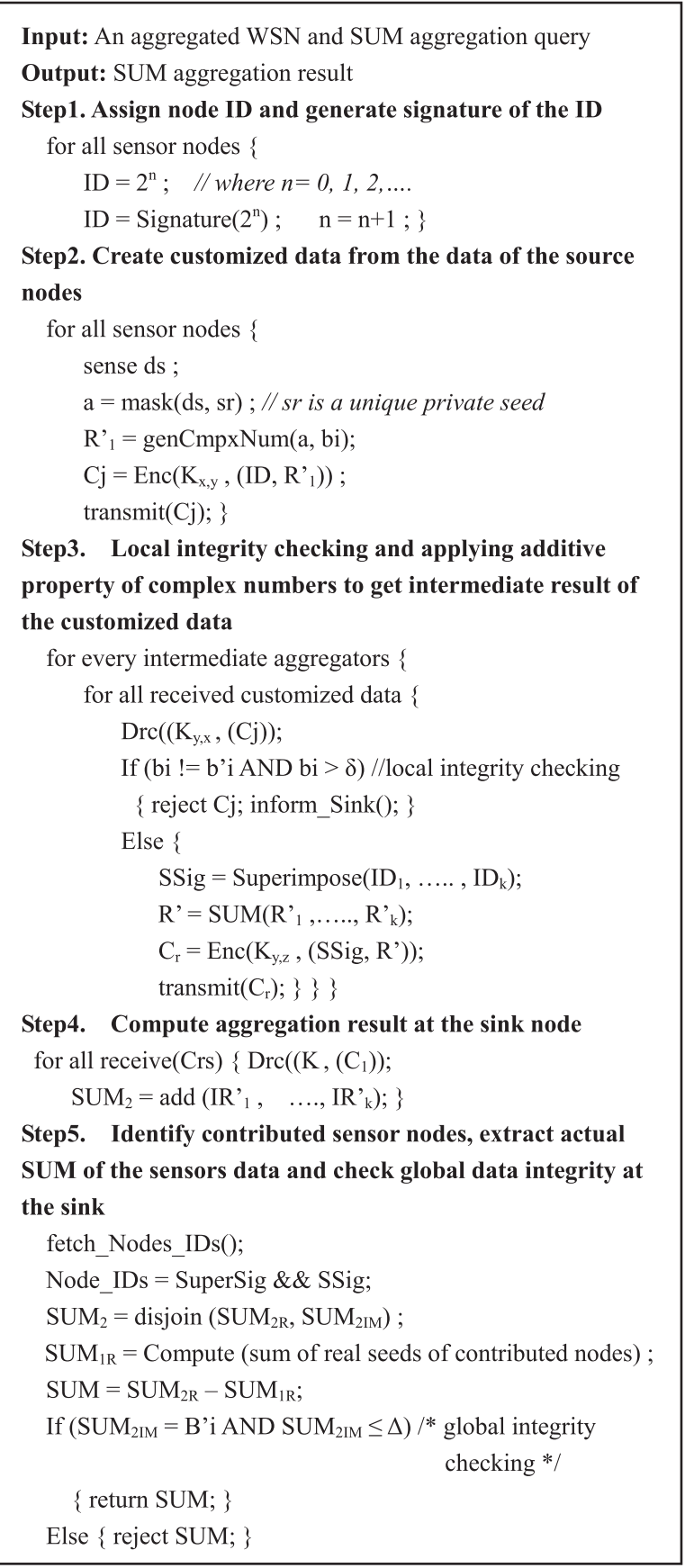

Fig. 2 Algorithm for SUM aggregation with privacy-preservation and integrity checking.

and communication bandwidth. Therefore, we must be careful to design an algorithm for WSNs so that the execution of the algorithm consumes as less resources as possible to run applications for longer time. In this section, we propose a new resource-efficient algorithm for SUM aggregation function for preserving data privacy with data integrity checking in WSNs. The algorithm that performs sensitive data aggregation and integrity checking is illustrated in Fig. 2. We explain five main steps of our algorithm as below.

In the first step, we assign a special type of positive in-

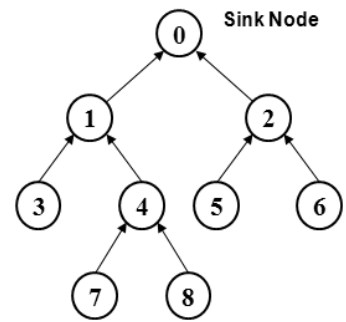

Fig. 3 Example of a multi-hop WSN.

Table 1 Real ID of 8 sensor nodes with signature.

\begin{tabular}{|l|l|l|}
\hline SN & \multicolumn{1}{|l|}{ Node-ID } & 2-byte Signature \\
\hline 1 & $2^{0}=1$ & 0000000000000001 \\
\hline 2 & $2^{1}=2$ & 0000000000000010 \\
\hline 3 & $2^{2}=4$ & 0000000000000100 \\
\hline 4 & $2^{3}=8$ & 0000000000001000 \\
\hline 5 & $2^{4}=16$ & 0000000000010000 \\
\hline 6 & $2^{5}=32$ & 0000000000100000 \\
\hline 7 & $2^{6}=64$ & 0000000001000000 \\
\hline 8 & $2^{7}=128$ & 0000000010000000 \\
\hline
\end{tabular}

teger $2^{n}$ (where, $n=0$ to $B n \times 8-1$, such that $B n$ is the number of free bytes available in the payload) to every sensor node as node ID as shown in Table 1 . The Table 1 is based on the Fig. 3 which is a multi-hop WSN consisting of eight nodes and a sink node. This is because the binary value of every integer of $2^{n}$ type has only one high bit (1). In addition, the position of the high bit for all integers of this type is unique. The sink node knows a data contributing sensor node through the signature of Node-ID. The Node-ID of a sensor node is used to generate a signature of a fixed length. A signature is a fixed size bit stream of binary numbers for a given integer. Signature of a senor node ID can be generated by using the technique presented in the work [19]. We can determine the length of the signature based on the size of a given WSN. When the size of the WSN increases we can increase the length of the signature up to the $B n$ bytes. In other words, different size WSNs can have signatures of different lengths. The detail of using signatures as node IDs has been presented in our previous work [18].

When the network receives a SQL like query for SUM aggregation function, in the second step, the sampled sensitive data $d s$ of each sensor node is, first, concealed in $a$ by combining with a unique seed ( $s r)$ which is a private real number. The seeds can be selected from an integer range (i.e., space between lower bound - upper bound). By increasing the size of the range, we can further increase the level of the data privacy. For instance, with a seed from the range of $\langle 1-10000\rangle$ can gives the probability of knowing the seed of a sensor node by other sensor node is 0.0001 . That is to say, data privacy-preserving efficacy, in terms of probability, for this range is 0.9999 . Similarly, a seed from the range of 1 - 20000 further improves the data privacy-preserving efficacy to 0.99995 . Hence, our approach can support data privacy feature strongly. To support data integrity, an integer 
Table 2 Customized data creation for each node.

\begin{tabular}{|c|c|c|c|c|c|}
\hline $\begin{array}{l}\mathbf{S} \\
\mathbf{N}\end{array}$ & $\begin{array}{c}\text { Rea- } \\
\text { ding } \\
\text { ds }\end{array}$ & $\begin{array}{c}\text { Real } \\
\text { Seed sr }\end{array}$ & $\begin{array}{c}\text { Mask } \\
\text { Value } \\
(\mathbf{a}=\mathbf{d s}+\mathbf{s r})\end{array}$ & $\begin{array}{c}\text { Difference } \\
\text { Value } \\
\text { bi }\end{array}$ & $\begin{array}{c}\text { Complex } \\
\text { Number } \\
(a+b i)\end{array}$ \\
\hline 1 & 16 & 40 & 56 & $2 \mathrm{i}$ & $56+2 i$ \\
\hline 2 & 14 & 51 & 65 & $0 \mathrm{i}$ & $65+0 \mathrm{i}$ \\
\hline 3 & 19 & 32 & 51 & $\mathrm{i}$ & $51+i$ \\
\hline 4 & 21 & 23 & 44 & $\mathrm{i}$ & $44+i$ \\
\hline 5 & 17 & 29 & 46 & $2 \mathrm{i}$ & $46+3 i$ \\
\hline 6 & 18 & 33 & 51 & $\mathrm{i}$ & $51+\mathrm{i}$ \\
\hline 7 & 13 & 39 & 52 & $2 \mathrm{i}$ & $52+2 i$ \\
\hline 8 & 15 & 67 & 82 & $2 \mathrm{i}$ & $82+2 i$ \\
\hline
\end{tabular}

value $b$ - the difference of the previous sensed value and the current sensed value of the sensor node- with $i$ is appended to the $a$ by using genCpxNum() function to form a complex number $C=a+b i$ as shown in Table 2. Based on Fig. 3 and Table 2, we illustrate data customization and signatures of all nodes in Fig. 4. For instance, the reading 16 of node 1 is changed into $56+2 i$. For this, the reading 16 is added to 40 which is a private seed of the node 1 to get a result 56. In addition, assuming that $2 \mathrm{i}$ is the difference value of previous reading and current reading of the node 1 , the $2 \mathrm{i}$ is appended to the result 56 to get $56+2 i$ which is a complex number form of the 16 after data customization process. The node 1 includes its signature, i.e., 00000001, when it transmits the data as: $\langle 00000001,56+2 i\rangle$. For the first round, the value of $b$ is zero. We assumed that any sensor node cannot be compromised before sending first round data to the sink node. Every source sensor node keeps the original sensed value $d$ of the current round to deduce $b$ in the next round which is updated in each round of data transmission. Next, the source node encrypts the customized data $R_{1}^{\prime}$, i.e., $R_{1}=a+b i$, and the signature of the node by using a key $\mathrm{K}_{\mathrm{x}, \mathrm{y}}$ (Sect. 3.2) and transmits the cipher text $C j$ to its parent. The term $K_{x, y}$ denotes a pairwise symmetric key shared by nodes $\mathrm{x}$ and $\mathrm{y}$ where the node $\mathrm{x}$ encrypts data by using a key $\mathrm{K}_{\mathrm{x}, \mathrm{y}}$ and the node $\mathrm{y}$ decrypts the data by using the key $\mathrm{K}_{\mathrm{x}, \mathrm{y}}$. In this way, our algorithm converts the sampled data into an encrypted complex number form. Hence, it not only protects the transmitting trend of private data but also doesn't let neighboring sensor nodes and adversaries to recover sensitive data even though they overheard and decrypted the sensitive data. This is the main principle of our scheme to preserve data privacy in WSNs.

In the third step, the parent sensor node (i.e., data aggregator) decrypts the received data by using respective pairwise symmetric keys of its child sensor nodes. For each child node, the parent node computes the difference value $\left(b^{\prime}\right)$ of the two real units by using the stored previous data and received current data of the child node. For the first round, the value of $b^{\prime}$ is also zero. For this, the parent node always keeps the record of the previously received data from each of the child nodes and it updates the previous data by

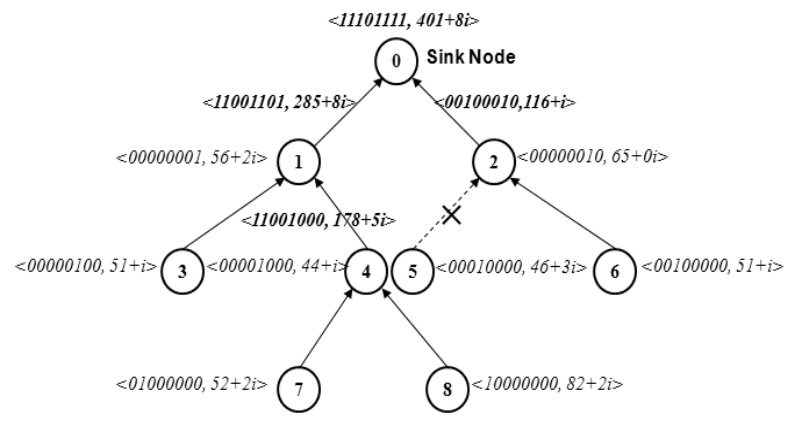

Fig. 4 Superimposing signatures and addition of customized sensor readings in a multi-hop WSN $(\delta=2)$.

current one in every round. To support local integrity checking, the parent node first compares just computed difference value with the currently received difference value (imaginary unit) from the child node and then compares the difference value with local threshold $\delta$. If the imaginary unit of the child's current data is equal to the computed difference value and the imaginary unit is not greater than $\delta$ then the parent node accepts the data of the child node. Otherwise, the parent node rejects the data of the child sensor node considering as polluted data. For example, we assume that the value for $\delta$ is set to 2 for local integrity checking. Because node 4 is the parent node of node 8 , the node 4 should check the local integrity of the node 8. In Fig. 4, since the imaginary part of node 8 's data is 2 which is less than or equals to $\delta$, node 4 accepts the data of node 8 . On the other hand, since the imaginary part of node 5's data is 3 , which is greater than $\delta$, its parent node 2 rejects the data of node 5 . In this way, the parent node assures the data integrity of child nodes. After that the parent node adds the data of child nodes including its own by using additive property of complex number to produce an intermediate result $R^{\prime}$. At the same time, it superimposes signatures (SSig) of the contributed nodes by performing bitwise OR operation on the bit-streams of the node IDs and forwards the encrypted intermediate result ' $\mathrm{C}_{\mathrm{r}}$ ' towards the sink node. Since this approach needs just one bit to carry an ID of a sensor node it is 16 times scalable than the existing work CMT [10] where plaintexts (2-byte each) are used for carrying IDs of sensor nodes by simply concatenating them. Note: Different types of application can have different value for the threshold $\delta$. For example, the body temperature of a patient cannot be changed by 5 -unit within some $10 \mathrm{~s}$ of seconds or a minute i.e., within an epoch (next round). On the other hand, consumption of electricity in a building can be changed, for example, by 10-unit within an epoch. Upper level sensor nodes i.e., data aggregators, always monitor such type of possible misbehavior of lower level sensor nodes. This situation happens only when an adversary compromises the child sensor node and pollutes the data. Thus, our algorithm supports local integrity checking which enforces to provide consistent data from child nodes. Above process continues at all nodes of the upper levels of the network until the whole partially aggregated data of the network reach to the sink node. 
In the fourth step, when the sink node receives all intermediate result sets $\mathrm{C}_{\mathrm{rs}}$ (partially aggregated encrypted customized data with superimposed signature) from the 1-hop child nodes, it decrypts them by using respective pairwise symmetric keys and computes the final aggregation $\mathrm{SUM}_{2}$ from $\mathrm{C}_{\mathrm{rs}}$. For instance, as shown in Fig. 4, when the sink node receives from node 1 and 2 data $\langle 11001101,285+8 \mathrm{i}\rangle$ and $\langle 00100010,116+\mathrm{i}\rangle$, respectively, it first decrypts the data of the two nodes by using the respective pairwise symmetric key shared with its counterpart and then combines their data into $\mathrm{SUM}_{2}=\langle 11101111,401+9 \mathrm{i}\rangle$ by superimposing the signatures. Since $S U M_{2}$ is of complex number form and the sensed data has been concealed in the real unit by using private seeds identifying the information of the contributed sensor nodes is necessary to deduce actual SUM value.

In the fifth and the last step, the sink node first knows data contributing nodes by checking the high bits (1s) of the received superimposed signature by performing bitwise AND operation with the pre-stored signature files or superimposed signature of the Node-IDs of the all nodes of the network. As shown in Fig. 4, for instance, node 5 is not contributing data because there is low bit (0) in its position on the signature. Then, it reverses the customization process of the second step. For this, it separates $S U M_{2}$ into real unit $S U M_{2 R}$ and imaginary unit $S U M_{2 I M}$. Because the sampled data of sensor nodes has been concealed within the real unit, the sink node computes the actual aggregated result $S U M$ by subtracting (an inverse operation of masking, step 2) $S U M_{1 R}$ (a freshly computed sum value of the private seeds of the contributed source nodes) from $S U M_{2 R}$. For example, in Table 2, the total aggregated value collected at the sink node is $447+12 \mathrm{i}$. The sink node separates $447+12 \mathrm{i}$ into 447 and $12 \mathrm{i}$. The value 447 is computed by combining the readings of all the nodes with their private seeds. Since the sink node stores the information of the private seeds of all nodes, the sink node can get the actual result, i.e., 133, by subtracting the sum of private seeds of all nodes (314) from the total aggregated value (447). The final result SUM is always accurate and reliable because of the following two reasons. First, a complex number is an algebraic expression and hence the underlying algebra gives the accurate result of the aggregated sensor data. Second, since the private seeds are fixed integer values (i.e., seeds are not random numbers) after collecting data by the sink node it subtracts exactly the same values that have been added to the sensor data during data hiding process by every source node.

At the same time, before accepting the $S U M$, the sink node performs global integrity checking of $S U M$ to assure whether the $S U M_{2}$ has been polluted by an adversary in transit or not. For this, like parent nodes, the sink node also computes the difference value $\left(B^{\prime}\right)$ of the two real units by using the stored previous data and received current data from the network. The sink node first compares just computed difference value $B^{\prime} i$ with the currently received difference value i.e., $S U M_{2 I M}$, from the network and then compares the difference value $\left(S U M_{2 I M}\right)$ with global threshold $\Delta$ (for every application, the maximum value for $\Delta=\delta \times N$, where $N$ is the total number of nodes in a network). If the imaginary unit $S U M_{2 I M}$ of the current data from the network is equal to the just computed difference value $B^{\prime} i$ and the $S U M_{2 I M}$ is not larger than $\Delta$ then the sink node accepts the data of the network and returned the actual $S U M$ to the query issuer. Otherwise, the sink node rejects the $S U M$ considering it as forged/polluted data by adversary or other nodes. For example, as shown in Fig. 4, we assume that a local integrity threshold per node $\delta$ equals to $2 i$ and the maximum value for a global threshold $\Delta$ can be computed as $\delta \times \mathrm{N}=2 i \times 8=16 i$. Since a sensor node N5 does not participate for data collection, the global integrity checking value $\Delta$ can be computed as $\delta \times \mathrm{N}=2 i \times 7=14 i$. In this scenario, the received data is considered as a consistent one and is accepted by the sink node because the value computed at the sink node, i.e., $9 i$, is the same as the one received from the network and the value is less than the global integrity checking value, i.e., $9 i<14 i$.

Using difference value of the previous and the current data to check data integrity is reasonable and justifiable because when a sensor node is compromised and even though it modifies the original data the compromised node can't change the previously sent data which has been already stored at the parent node. In such scenario, there occurs mismatch between imaginary unit coming from a child node and the just computed difference value at the parent node because the imaginary unit is also the difference value of the two real units of the previous data and the current data of the node. Hence, our method can identify and exclude the adversary at the cost of $O(1)$ round as compared to the conventional cost of $O(\log N)$ rounds [5] which selects a different portion of the sensor nodes that participate in the aggregation where $N$ is the total number of sensor nodes in a WSN. In this way, the propose method can provide local data integrity checking and global data integrity checking at the data aggregators/parent nodes and the sink node respectively. Our method needs a few arithmetic operations (additions and subtractions) for assuring data integrity and only 2-byte (for sending difference value) is the communication overhead to achieve data integrity checking.

\subsection{Analysis}

The energy consumption issue of WSNs is the most important because the lifetime of a sensor node is extremely dependent on the available energy of its battery. Preserving data privacy and supporting integrity checking consume a significant amount of resources adding extra communication and computation overheads to the WSNs. In addition, data propagation delay is critical for applications like collecting patients' health data. Such delay also consumes energy of the nodes because nodes have to run for longer time. To address these problems, in this section, we design analytical models in order to show the resources-efficiency of our method to aggregate sensitive data protecting data integrity. There are three metrics which directly affect the lifetime of a WSN. They are communication (message) overhead, 
Table 3 Summary of the analytical models of privacy preservation and integrity protection.

\begin{tabular}{|c|c|c|c|c|c|c|}
\hline \multirow{2}{*}{ Scheme } & \multirow{2}{*}{ Node Type } & \multicolumn{3}{|c|}{ Computation Overhead } & \multirow{2}{*}{$\begin{array}{c}\text { Communication } \\
\text { Overhead }\end{array}$} & \multirow{2}{*}{$\begin{array}{c}\text { Data Propagation } \\
\text { Delay }\end{array}$} \\
\hline & & Arithmetic Operations & Encryption & Decryption & & \\
\hline \multirow{2}{*}{ iPDA } & Leaf Node & $6(-), 5(+)$ & 5 & 5 & \multirow{2}{*}{$\mathrm{O}(6)$} & \multirow{2}{*}{$6 \times \mathrm{DC}$} \\
\hline & Aggregator & $6(-), 7(+)$ & 5 & 5 & & \\
\hline \multirow{2}{*}{ iCPDA } & Cluster Head & $\begin{array}{l}\text { Addition of three 2-polynomials } \\
\text { and Inverse and multiplication of }\end{array}$ & 2 & 4 & \multirow{2}{*}{$\mathrm{O}(4)$} & \multirow{2}{*}{$4 \times \mathrm{DC}$} \\
\hline & Cluster Member & & 3 & 2 & & \\
\hline \multirow{2}{*}{$\begin{array}{c}\text { Our } \\
\text { Scheme }\end{array}$} & Leaf Node/CM & $1(-), 2(+), 1(\times)$ & 1 & 0 & \multirow{2}{*}{$\mathrm{O}(1)$} & \multirow{2}{*}{ DC } \\
\hline & Aggregator/CH & $3(-), 6(+), 1(\times)$ & 1 & 2 & & \\
\hline
\end{tabular}

computation overhead (the burden to sensor processor), and data propagation delay (average time taken by the sampled data to reach the sink node). Due to the lack of the space, we summarize the analytical models as shown in the Table 3. Data propagation delay is presented in terms of duty cycling (DC) which is the time required by a sensor node to sample, receive, process and transmit the data (i.e., one round communication).

\section{Performance Evaluation}

The energy consumption issue of WSNs is the most important one because the lifetime of a sensor node is extremely dependent on the available energy of its battery. Although sensing activity, communication and data processing are three domains to be considered for energy consumption, the communication overhead is responsible for the bulk of the energy consumption [3] in a WSN. In this section, we present simulation results of our scheme by comparing it with iPDA and iCPDA schemes in terms of communication overhead and data propagation delay and integrity checking. For this, we use TOSSIM [13] simulator running over TinyOS [20] operating system and GCC compiler. We consider 100 sensor nodes distributed randomly in $100 \mathrm{~m} \times$ $100 \mathrm{~m}$ area. As presented in directed diffusion [9], we use such parameters as receiving power dissipation of $395 \mathrm{~mW}$ and transmitting power dissipation of $660 \mathrm{~mW}$. Moreover, MATLAB $^{\circledR}$ 7.6.0.324 (R2008a) is used to get execution time required for data customization and data aggregation.

Figure 5 shows communication overhead in terms of the number of messages generated in a WSN with respect to varying number of sensor nodes. As expected, the number of messages in the iPDA, iCPDA and our schemes increases when the number of sensor nodes increases. This is because every sensor node in the WSN is capable of sensing data and when the number of source nodes increases, the number of messages also naturally increases in all of the three schemes. However, our scheme outperforms the iPDA and iCPDA schemes because the existing schemes generate unnecessary messages in the network. The reason is that in our scheme each sensor node can customize its data by itself and it doesn't need to generate extra messages in the network for data privacy and integrity checking. On the other hand,

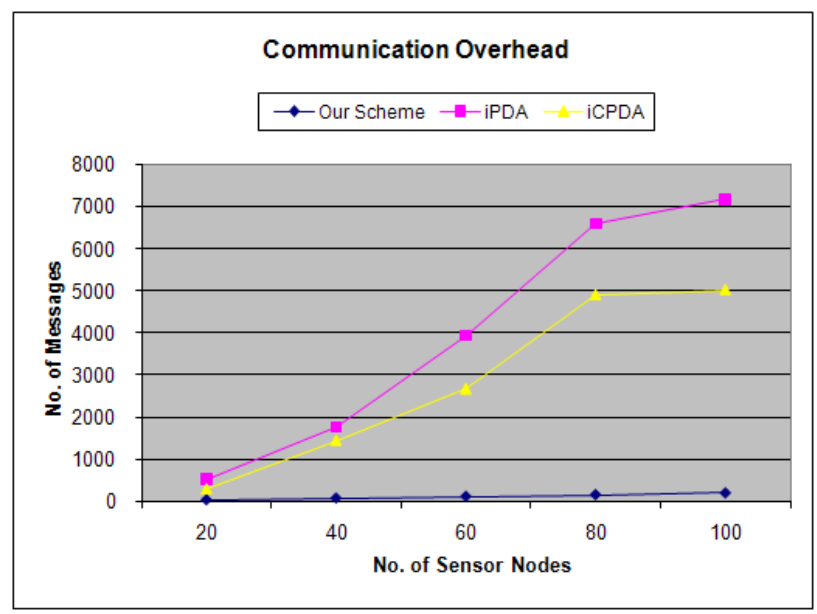

Fig. 5 Number of messages generated by the iPDA, iCPDA and our schemes.

the iPDA and iCPDA schemes generate six messages and four messages, respectively, for privacy preservation and integrity checking. Due to many messages exchanged among the nodes, there occur high data collisions in the existing schemes. Because of this, in the existing schemes, the number of messages generated in the network increases greatly for successful data transmissions. Therefore, the iPDA and iCPDA schemes are very expensive in terms of communication overhead than our scheme.

The messages generated in the WSN are finally consumed by the sink node. For this, message transmission and message reception processes are involved. Both processes require significant amount of energy. Figure 6 shows communication overhead in terms of energy dissipation by the iPDA, iCPDA and our schemes with respect to varying number of sensor nodes in the WSN. As expected, the dissipated energy by all three schemes increases when the number of sensor nodes increase. This is because every message generated in the network requires some amount of energy to reach the sink node. However, the power consumption by our scheme is always lower than that of iPDA and iCPDA schemes. The reason is that the iPDA and iCPDA schemes generate too many unnecessary messages in the WSN while achieving integrity protecting and privacy preservation in 


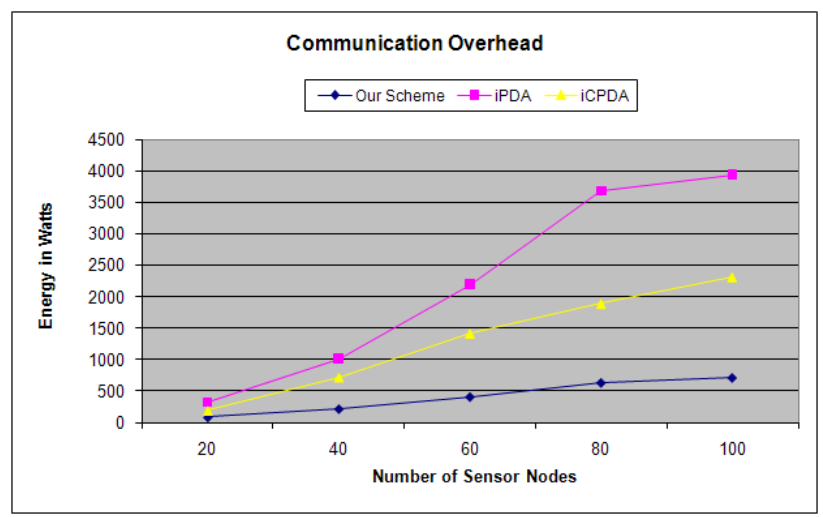

Fig. 6 Energy consumption by the iPDA, iCPDA and our schemes.

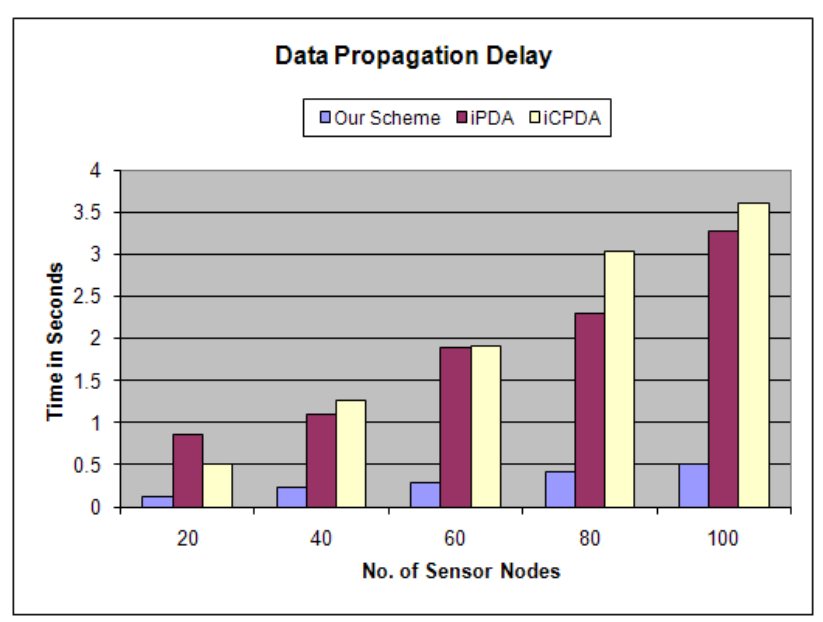

Fig.7 Average data transmissions time for iPDA, iCPDA and our schemes.

data aggregation. And also every sensor node becomes active for longer time (longer a node becomes active more the energy requires) to communicate all the messages.

Figure 7 shows data propagation delay in terms of average time required by sampled data of sensor nodes to reach to the sink node considering data privacy and integrity checking. During this process, a sensor node in iPDA and iCPDA has to communicate (i.e., transmit and receive) at least six and four messages respectively as compared with our scheme's one message. Hence, sensor nodes in both iPDA and iCPDA need more active time to perform all communications than our scheme resulting very high data propagation delay in the existing work. In this way, duty-cycling is also increased in the existing schemes. The iCPDA generates less number of messages than the iPDA but has complex computation for privacy preservation and longer size message than that of the iPDA. Moreover, in iCPDA, the sampled data of sensor nodes is sent to the opposite direction (data is transmitted from the cluster head to the cluster members) of the sink node for privacy preservation process. Therefore, the iCPDA has the worst performance among the three schemes.

When adversaries manipulate messages in the network

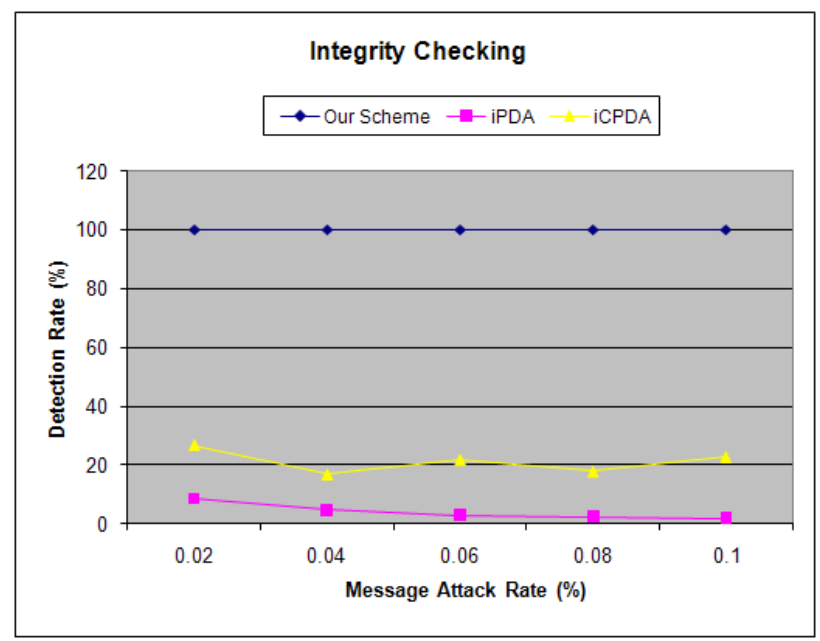

Fig. 8 Integrity checking by iPDA, iCPDA and our schemes when some messages are polluted.

Table 4 Computational overhead for data customization and aggregation.

\begin{tabular}{|c|c|}
\hline Protocols & Execution Time (in Secs.) \\
\hline iPDA & 0.005924 \\
\hline iCPDA & 0.219325 \\
\hline Our Scheme & 0.002632 \\
\hline
\end{tabular}

it is required to detect them. Figure 8 compares integrity checking feature of all the three schemes. It is shown that our scheme can detect every polluted message but the iPDA and iCPDA has very low rate of polluted message detection. The reason is that every node in our scheme performs local integrity checking of the coming data from the lower level nodes. But, only sink node checks the integrity in iPDA and so does the cluster heads in iCPDA.

Table 4 shows computation overhead of data customization and data aggregation processes. The result shows that the iCPDA has the worst performance to aggregate data by preserving data privacy. The reason is that the iCPDA uses a complex computation method to achieve data privacy. On the other hand, the computation cost of our scheme is about two times faster than that of the iPDA. Our scheme and the iPDA are about 83 and 35 times faster than the iCPDA respectively. It means that both iPDA and our scheme reduce a significant amount of resource (CPU time) usage to achieve private data aggregation.

\section{Conclusions and Future Work}

In this paper, we proposed an efficient and general scheme in order to aggregate sensitive data protecting data integrity for private data generating environments such as patients' health monitoring application. For maintaining data privacy, our scheme applies the additive property of complex numbers where sampled data are customized and given the form of complex number before transmitting towards the sink node. As a result, it protects the trend of private data of 
a sensor node from being known by its neighboring nodes including data aggregators in WSNs. Moreover, it is still difficult for an adversary to recover sensitive information even though data are overheard and decrypted. Meanwhile, data integrity is protected by using the imaginary unit of complex-number-form customized data at the cost of just two extra bytes.

Our scheme is built on the top of an existing efficient key management scheme. In addition, we provide a method for data privacy by using complex numbers form. Therefore, we believe that both of them work cooperatively to provide the basic security properties like access control, message integrity, message confidentiality and data privacy as well.

Through simulation results, we have shown that our scheme is much more efficient in terms of communication and computation overheads, data propagation delay and integrity checking than the IPDA and ICPDA schemes. As future work, we will provide more simulation results by designing data integrity and sensitive data preserving scheme under collusive attacks.

\section{Acknowledgements}

This research was supported by Basic Science Research Program through the National Research Foundation of Korea (NRF) funded by the Ministry of Education, Science and Technology (grant number 2010-0023800). And this research was also financially supported by the Korean Research Foundation Grant funded by Korean Government (MOEHRD) (KRF-2007-211-D00103).

\section{References}

[1] J. Yick, B. Mukherjee, and D. Ghosal, "Wireless sensor network survey," Comput. Netw., vol.52, no.12, pp.2292-2330, 2008.

[2] J. Considine, F. Li, G. Kollios, and J. Byers, "Approximate aggregation techniques for sensor databases," Proc. ICDE, April 2004.

[3] Madden, R. Samuel, Franklin, J. Michael, Hellerstein, M. Joseph, and W. Hong, "TAG: A tiny aggregation service for ad hoc sensor networks," OSDI, 2002.

[4] Madden, R. Samuel, Franklin, J. Michael, Hellerstein, and M. Joseph, "TinyDB: An acquisitional query processing system for sensor networks," ACM Trans. Database Syst., vol.30, no.1, pp.122173.

[5] W. He, X. Liu, H. Nguyen, K. Nahrstedt, and T. Abdelzaher, "PDA: Privacy-preserving data aggregation in wireless sensor networks," Proc. 26th IEEE International Conference on Computer Communications, pp.2045-2053, May 2007.

[6] Y. Yang, X. Wang, and S. Zhu, "SDAP: A secure hop-by-hop data aggregation protocol for sensor networks," Proc. 7th ACM Interational Symposium on Mobile Ad Hoc Networking and Computing, May 2006.

[7] W. He, X. Liu, H. Nguyen, K. Nahrstedt, and T. Abdelzaher, "iPDA: An integrity-protecting private data aggregation scheme for wireless sensor networks," IEEE MILCOM, pp.1-7, Nov. 2008.

[8] E. Mlaih and S.A. Aly, "Secure hop-by-hop aggregation of end-toend concealed data in wireless sensor networks," IEEE INFOCOM Workshops, pp.1-6, April 2008.

[9] C. Itanagonwiwat, R. Govindan, and D. Estrin, "Directed diffusion: A scalable and robust communication paradigm for sensor networks," MobiCom, 2002.
[10] C. Castelluccia, E. Mykletun, and G. Tsudik, "Efficient aggregation of encrypted data in wireless sensor networks," The Second Annual International Conference on Mobile and Ubiquitous Systems: Networking and Services, pp.109-117, 2005.

[11] M. Horton, D. Culler, K. Pister, J. Hill, R. Szewczyk, and A. Woo, "MICA the commercialization of micro sensor motes," Sensors, vol.19, no.4, pp.40-48, April 2002.

[12] E.-O. Blaß and M. Zitterbart, "An efficient key establishment scheme for secure aggregating sensor networks," Proc. 2006 ACM Symposium on Information, Computer and Communications Security, pp.303-310, March 2006.

[13] P. Levis, N. Lee, M. Welsh, and D. Cullar, "TOSSIM: Accurate and scalable simulation of entire TinyOS applications," http://www.cs.berkely.edu/pal/research/tossim.html

[14] R. Bista, Y.K. Kim, and J.W. Chang, "A new approach for energybalanced data aggregation in wireless sensor networks," CIT09, cit, vol.2, pp.9-15, 2009.

[15] T. Feng, C. Wang, W. Zhang, and L. Ruan, "Confidentiality protection for distributed sensor data aggregation," Proc. 27th IEEE International Conference on Computer Communications, pp.475-483, 2008.

[16] M. Conti, L. Zhang, S. Roy, R. Di Pietro, S. Jajodia, and L-V. Mancini, "Privacy-preserving robust data aggregation in wireless sensor networks," Security Comm. Networks, vol.2, pp.195-213, 2009.

[17] W. He, X. Liu, H. Nguyen, and K. Nahrstedt, "A cluster-based protocol to enforce integrity and preserve privacy in data aggregation," Proc. 29th IEEE International Conference on Distributed Computing Systems Workshops, pp.14-19, 2009.

[18] R. Bista and J.W. Chang, "Energy efficient data aggregation for wireless sensor networks," Sustainable Wireless Sensor Networks, 2010, ISBN: 978-953-307-297-5, InTech, Available from: http://www.intechopen.com/articles/show/title/ energy-efficient-data-aggregation-for-wireless-sesor-networks

[19] J. Zobel, A. Moffat, and K. Ramamohanarao, "Inverted files versus signature file for text indexing," ACM TDS, vol.23, no.4, pp.453490, 1998.

[20] J. Hill, R. Szewczyk, A. Woo, S. Hollar, D.E. Culler, and K.S.J. Pister, "System architecture directions for networked sensors," ASPLOS'02, pp.93-104. TinyOS is available at http://webs.cs.berkeley.edu

[21] G.S. Simmons, "An introduction to shared secret and/or shared control schemes and their application," Contemporary Cryptology, IEEE Press, 1992.

[22] L. Eschenauer and V.D. Gligor, "A key-management scheme for distributed sensor networks," Proc. 9th ACM Conference on Computer and Communications Security, pp.41-47, Nov. 2002.

[23] R.L. Rivest, "The RC5 encryption algorithm," Proc. 1994 Leuven Workshop on Fast Software Encryption, pp.86-96, Springer, 1995.

[24] H.M. Sun and S.-P. Shieh, "Constructing perfect secret sharing schemes for general and uniform access structures," J. Information Science and Engineering, vol.15, pp.679-689, 1999. 


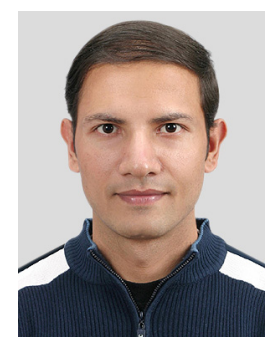

Rabindra Bista received the B.Sc. degree in Information Technology from Sikkim Manipal University, India, in 2004. He received the M.S. and Ph.D. degrees in the Computer Engineering from Chonbuk National University in 2007 and 2011, respectively. Currently, he is working as a Faculty in Computer Science and Engineering Department at Kathmandu University, Nepal. His research interests include spatial network database, middleware, wireless sensor networks and software engineering.

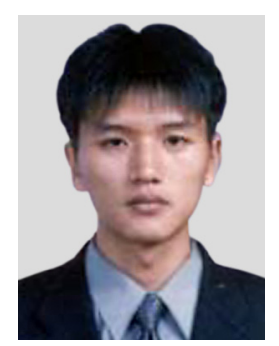

Yong-Ki Kim received the B.S., M.S. and Ph.D. degrees in Computer Engineering from Chonbuk National University in 2002, 2005 and 2011, respectively. Currently, he is working as a Post Doc. at Chonbuk National University. His research interests include spatial network database, query processing and sensor network.

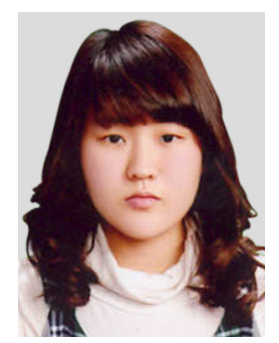

Myoung-Seon Song received the B.S. degree in Computer Engineering from Chonbuk National University in 2010. She is a candidate of M.S. degree in the Computer Engineering Department of the same university since 2010 . Her research interests include spatial network database, query processing and sensor network.

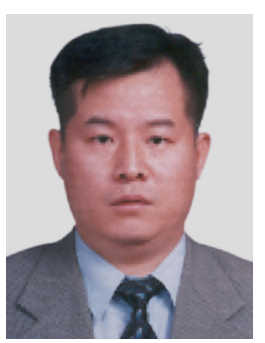

Jae-Woo Chang is a professor in the Department of Computer Engineering, Chonbuk National University, Korea since 1991. He received the B.S. degrees in Computer Engineering from Seoul National University in 1984. He received the M.S. and Ph.D. degrees in Computer Engineering from Korea Advanced Institute of Science and Technology (KAIST) in 1986 and 1991, respectively. During 1996-1997, he stayed in University of Minnesota for visiting scholar. And during 2003-2004, he worked for Penn State University (PSU) as a visiting professor. His research interests include spatial network database, context awareness and storage system. 